\title{
A Patient with Non-alcoholic Steatohepatitis Complicated by Multiple Myeloma
}

\author{
Momoko Akashi ${ }^{1}$, Kazuto Tajiri ${ }^{1}$, Akinori Wada $^{2}$, Koichi Tsuneyama ${ }^{3}$, Kengo Kawai ${ }^{1}$, \\ Satoshi Yasumura ${ }^{1}$, Masami Minemura ${ }^{1}$, Terumi Takahara ${ }^{1}$ and Toshiro Sugiyama ${ }^{1,2}$
}

\begin{abstract}
:
A 68-year-old woman with liver dysfunction was diagnosed with nonalcoholic steatohepatitis (NASH) stage 1. Three years later, she showed massive ascites and jaundice. A trans-jugular liver biopsy confirmed advanced cirrhosis, suggesting that her liver fibrosis had progressed rapidly. At the same time, she was diagnosed with multiple myeloma (MM). In this case, the plasma levels of osteopontin (OPN), a proinflammatory cytokine that promotes liver fibrosis progression through the hedgehog pathway and is increased in patients with MM, were increased. This increased OPN expression was accompanied by the upregulation of the hedgehog pathway in this patient, suggesting that the MM-associated increase in OPN had promoted the progression of liver fibrosis through the hedgehog pathway. The progression of liver fibrosis should be monitored in patients with NASH if other diseases, such as MM, are present.
\end{abstract}

Key words: non-alcoholic steatohepatitis, multiple myeloma, osteopontin, hedgehog pathway

(Intern Med 57: 2013-2018, 2018)

(DOI: 10.2169/internalmedicine.0092-17)

\section{Introduction}

Nonalcoholic fatty liver disease (NAFLD) is the most common liver disease and the leading cause of cirrhosis in developed countries. About 20-30\% of patients with NAFLD develop nonalcoholic steatohepatitis (NASH) (1), with 5$20 \%$ of the latter progressing to cirrhosis within 5-10 years (2). Risk factors for fibrosis progression in patients with NASH include diabetes, obesity, age and a high degree of inflammation on the initial liver biopsy $(3,4)$.

Osteopontin (OPN), an extracellular matrix protein that acts as a proinflammatory cytokine, is important in bone resorption, inflammation, angiogenesis and fibrosis in various tissues. OPN expression is increased in patients with multiple myeloma (MM), a bone-resorbing disease, with the degree of increase correlating with the severity of MM (5-9). In NASH, OPN has been reported to promote liver fibrosis progression through the hedgehog pathway $(10,11)$.

We encountered a patient with NASH who showed rapid fibrosis progression after developing MM. This report de- scribes the clinical course of this patient, showing that increased OPN due to MM results in rapid liver fibrosis progression in patients with NASH.

\section{Case Report}

A 63-year-old Japanese woman presented with liver dysfunction and underwent a percutaneous liver biopsy to evaluate the etiology of this dysfunction. Laboratory findings showed an alanine aminotransferase (ALT) dominant liver injury without jaundice (in parentheses, Table 1). She was asymptomatic, with a preserved platelet count (18.6x $\left.10^{4} / \mu \mathrm{L}\right)$ and serum albumin concentration $(4.7 \mathrm{~g} / \mathrm{dL})$. A liver biopsy showed fat deposits of various sizes in the hepatic lobe, hepatocyte ballooning and pericellular fibrosis (Fig. 1), with slight infiltration of inflammatory cells into the liver. She was not infected with the hepatitis virus and did not have autoantibodies. She was obese (body mass index 27.7 $\mathrm{kg} / \mathrm{m}^{2}$ ), with no alcohol intake. She was not taking any medications, including herbal remedies and supplements. These findings resulted in a diagnosis of NASH (Brunt clas-

${ }^{1}$ Department of Gastroenterology, Toyama University Hospital, Japan, ${ }^{2}$ Department of Hematology, Toyama University Hospital, Japan and ${ }^{3}$ Department of Pathology and Laboratory Medicine, Graduate School of Biomedical Sciences, Japan

Received: August 20, 2017; Accepted: December 19, 2017; Advance Publication by J-STAGE: February 28, 2018

Correspondence to Dr. Kazuto Tajiri, tajikazu@med.u-toyama.ac.jp 
Table 1. Laboratory Findings at the Second Liver Biopsy (at the Initial Biopsy).

\begin{tabular}{lrlc}
\hline$<$ Blood chemistry> & & $<$ Complete blood count & \\
Total protein & $5.4(7.2) \mathrm{g} / \mathrm{dL}$ & White blood cells & $2.97(5.61) \times 10^{3} / \mu \mathrm{L}$ \\
Albumin & $3.2(4.7) \mathrm{g} / \mathrm{dL}$ & Red blood cells & $273(411) \times 10^{4} / \mu \mathrm{L}$ \\
Aspartate aminotransferase & $109(104) \mathrm{IU} / \mathrm{L}$ & Hemoglobin & $9.8(13.1) \mathrm{g} / \mathrm{dL}$ \\
Alanine aminotransferase & $60(198) \mathrm{IU} / \mathrm{L}$ & Hematocrit & $28.3(39.1) \%$ \\
Lactate dehydrogenase & $319(239) \mathrm{IU} / \mathrm{L}$ & Platelets & $12.2(18.6) \times 10^{4} / \mu \mathrm{L}$ \\
Alkaline phosphatase & $603(380) \mathrm{IU} / \mathrm{L}$ & & \\
$\gamma$-glutamyl transpeptidase & $269(57) \mathrm{IU} / \mathrm{L}$ & $<$ Coagulation> & \\
Total-bilirubin & $2.4(0.8) \mathrm{mg} / \mathrm{dL}$ & Prothrombin activity & $56(89) \%$ \\
Blood urea nitrogen & $6(13) \mathrm{mg} / \mathrm{dL}$ & & \\
Creatinine & $0.5(0.5) \mathrm{mg} / \mathrm{dL}$ & $<$ Urinalysis $>$ & \\
Calcium & $8.4(9.4) \mathrm{mg} / \mathrm{dL}$ & Bence-Jones protein & positive (n.e.) \\
$<$ Serological tests $>$ & & & $0.69 \mathrm{mg} / \mathrm{dL}$ \\
Immunoglobulin G & $1,16(1,069) \mathrm{mg} / \mathrm{dL}$ & C-reactive protein & negative (negative) \\
Immunoglobulin A & $76(56) \mathrm{mg} / \mathrm{dL}$ & Hepatitis B surface antigen & negative (negative) \\
Immunoglobulin M & $16(18) \mathrm{mg} / \mathrm{dL}$ & Hepatitis B core antibody & negative (negative) \\
M protein & $(-)$ & Hepatitis C virus antibody & negative (negative) \\
Hyaluronic acid & $843(104) \mathrm{ng} / \mathrm{mL}$ & Antinuclear antibody & 3.7 (n.e.) mg/L \\
Type IV collagen & $13.2($ n.e. $) \mathrm{ng} / \mathrm{mL}$ & $\beta 2$-microglobulin & \\
\hline n.e.: not examined & & &
\end{tabular}

a

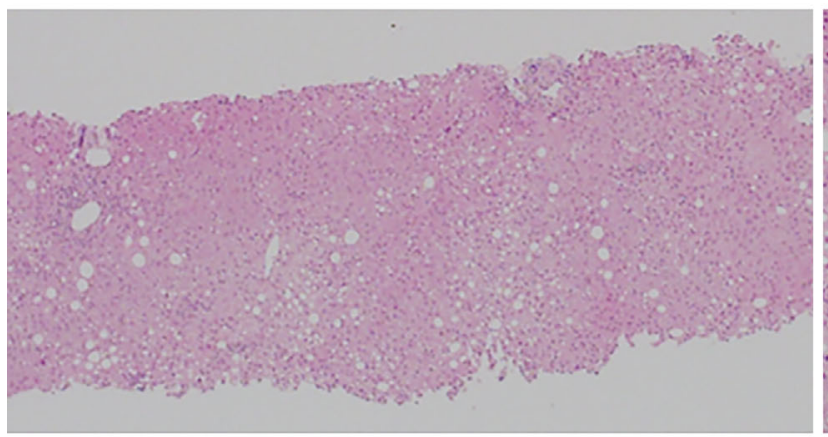

C

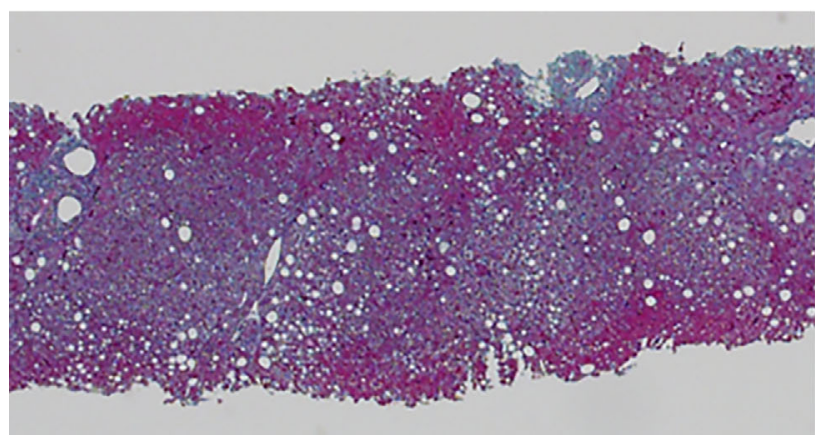

b

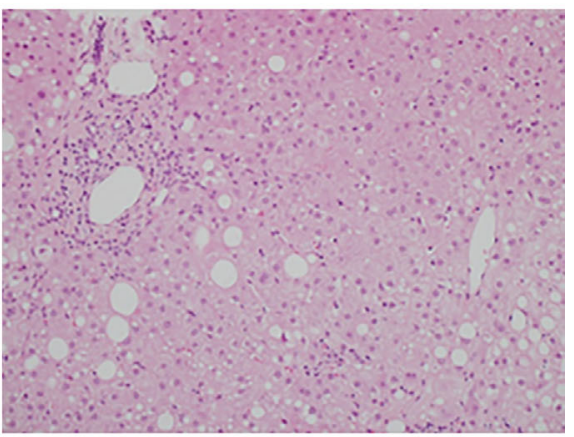

d

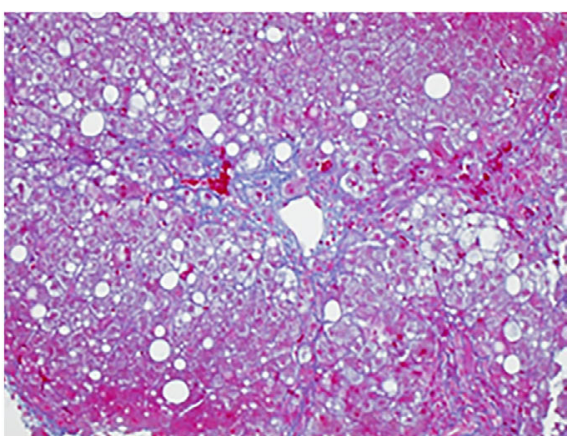

Figure 1. A histological examination of the liver at the initial diagnosis of NASH. Many lipid droplets and hepatocyte ballooning were present (a, b), as was pericellular fibrosis (c, d). The patient was histologically diagnosed with grade 1 and stage 1 NASH according to the Brunt classification (12). (a, b) Hematoxylin and Eosin staining, original magnifications: (a) 10×, (b) 40×; (c, d) Azan staining,

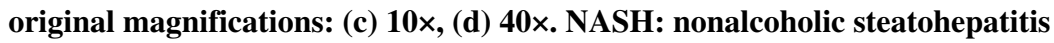

sification: grade 1, stage 1; NAS score: 5) $(12,13)$, and she was regularly followed thereafter.

Three years later, she complained of abdominal fullness and general malaise. Her body weight had not changed over this period. A physical examination revealed ascites and lower leg edema. Blood tests showed pancytopenia, liver dysfunction and elevated fibrosis markers (Table 1). Her blood cell counts had decreased over the previous year. Dy- 
a

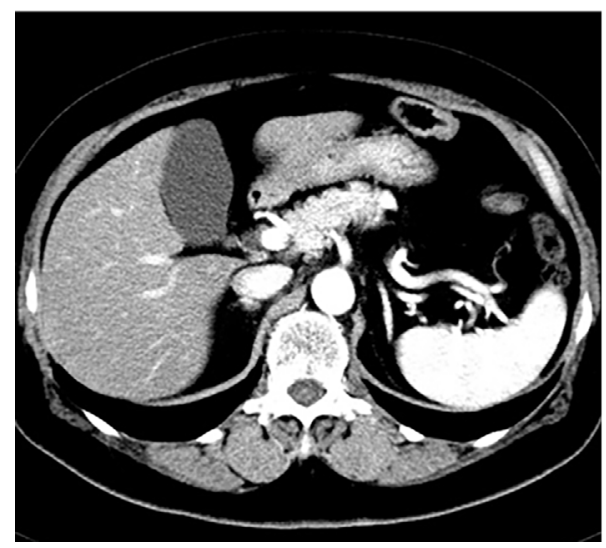

b

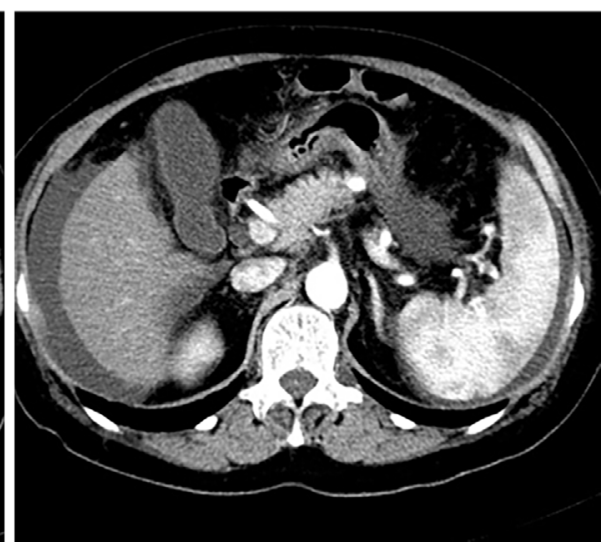

Figure 2. Abdominal computed tomography scans. Although ascites, liver atrophy and splenomegaly were not observed at the initial examination (a), these findings were observed three years later (b).

namic computed tomography of the abdomen showed liver cirrhosis, splenomegaly and ascites, findings not observed three years earlier (Fig. 2). She was negative for hepatocellular carcinoma. Her ascites were leaky, but pathogenic bacteria and malignant cells were not detected. She was therefore diagnosed with cirrhosis progressing from NASH. Because the fibrosis progression was rapid, we investigated whether or not other diseases responsible for cirrhosis and a deteriorating liver function, such as autoimmune hepatitis or amyloidosis, were present. However, autoantibodies were not detected, and there was no evidence of amyloid deposition on endoscopy or echocardiography. A liver biopsy obtained through the transjugular approach showed severe fibrosis and a decrease in lipid droplets compatible with burnout NASH (Brunt classification: grade 3, stage 4; NAS score: 6) (Fig. 3a and b). Congo red staining showed no amyloid deposition in the liver (Fig. 3c). Despite having cirrhosis, this patient showed no increase in the immunoglobulin (Ig) $\mathrm{G}$ concentration (Table 1) or decreases in IgA and IgM concentrations, suggesting that immunoglobulin production was inhibited. Immunoelectrophoresis showed an absence of Mprotein, but an immunofixation analysis showed the presence of Bence-Jones protein (BJP). Bone marrow aspiration showed an increase in plasma cells to $49.4 \%$, and immunostaining and in situ hybridization showed $\operatorname{Ig} k$ chain restriction (data not shown). The patient was therefore diagnosed with MM (BJP kappa type, International staging system: stage II, Durie \& Salmon staging: Stage IIA) accompanied by NASH-cirrhosis $(14,15)$. She did not have any bone lesions. We retrospectively estimated that MM had developed at least one year before the second liver biopsy, as the progression of anemia had appeared.

Because her only symptom of MM was anemia and her liver function was decreased, she was treated for MM with low doses of dexamethasone (20 mg/day) once a week. Following treatment, the number of plasma cells in her bone marrow had not increased. She was also treated for decompensated cirrhosis with diuretics, branched-chain amino acids and lactulose, depending on her symptoms. She was dis- charged after her condition stabilized. Two years after being diagnosed with MM (5 years after being initially diagnosed with NASH), she developed spontaneous bacterial peritonitis on two occasions, resulting in the cessation of dexamethasone. She experienced gradual progression of liver and renal failure and died six years after the initial diagnosis of NASH.

To assess whether or not MM was involved in NASHassociated fibrosis progression, we measured her plasma concentration of OPN, using a commercially available enzyme-linked immunosorbent assay kit according to the manufacturer's instructions (Immuno-Biological Laboratories, Gunma, Japan). Furthermore, NASH-related cirrhosis was thought to be associated with activation of the hedgehog pathway $(10,16)$. Hedgehog ligand family members [Sonic Hedgehog, Indian Hedgehog (IHH) and Desert Hedgehog] activate hedgehog signaling by engaging the receptor on the surface of hedgehog-responsive cells (11), which results in the nuclear localization of hedgehogregulated transcription factors, Glioblastoma (GLI) family (GLI1, GLI2 and GLI3). Therefore, the expression of IHH and GLI2 indicate activation of the hedgehog pathway. To detect the activation of the hedgehog pathway in the liver, we immunostained tissue samples with polyclonal antibodies against the zinc finger protein GLI2 (Aviva Systems Biology, San Diego, USA) and IHH protein (Aviva Systems Biology). As a result, at the time she was diagnosed with NASH-cirrhosis and MM, her plasma concentration of OPN was $1,970 \mathrm{ng} / \mathrm{mL}$, about 6 -fold higher than the concentration of $309 \pm 34 \mathrm{ng} / \mathrm{mL}$ in healthy controls (8). This was much higher than that with NASH cirrhosis without MM, which was measured in other three patients with NASH-cirrhosis at our hospital. Her plasma OPN level decreased to $838 \mathrm{ng} / \mathrm{mL}$ 2 years after dexamethasone treatment, but it again increased to $1,930 \mathrm{ng} / \mathrm{mL}$ after the cessation of dexamethasone treatment (Table 2). Immunostaining showed that GLI2 and IHH were absent from the first liver biopsy sample (Fig. 4a and b) but were overexpressed in the second biopsy sample at the portal area and periportal hepatocytes, sug- 


\section{a}

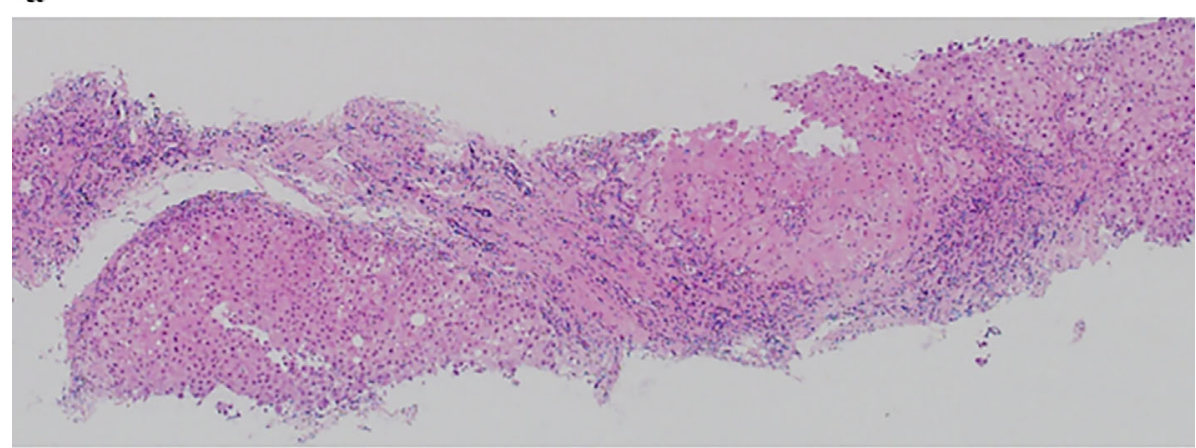

$\mathrm{b}$

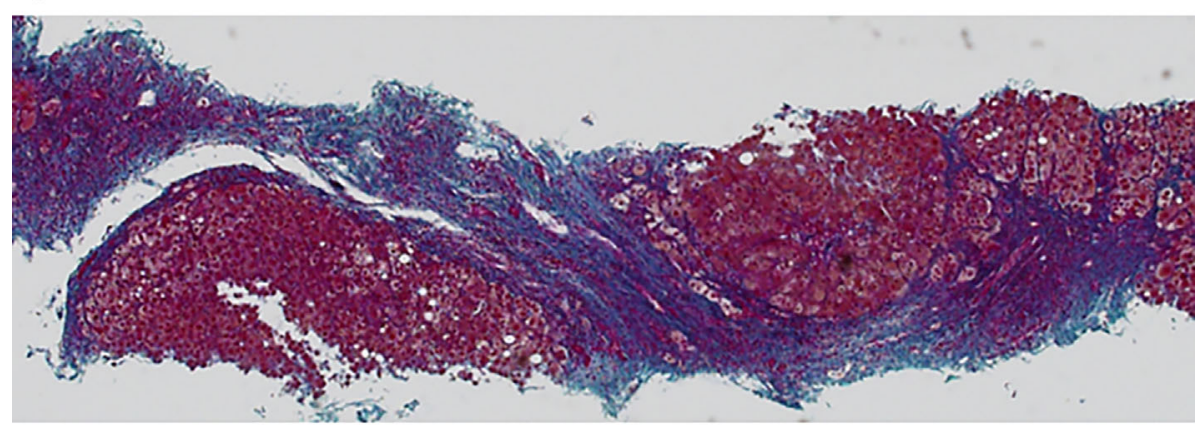

C

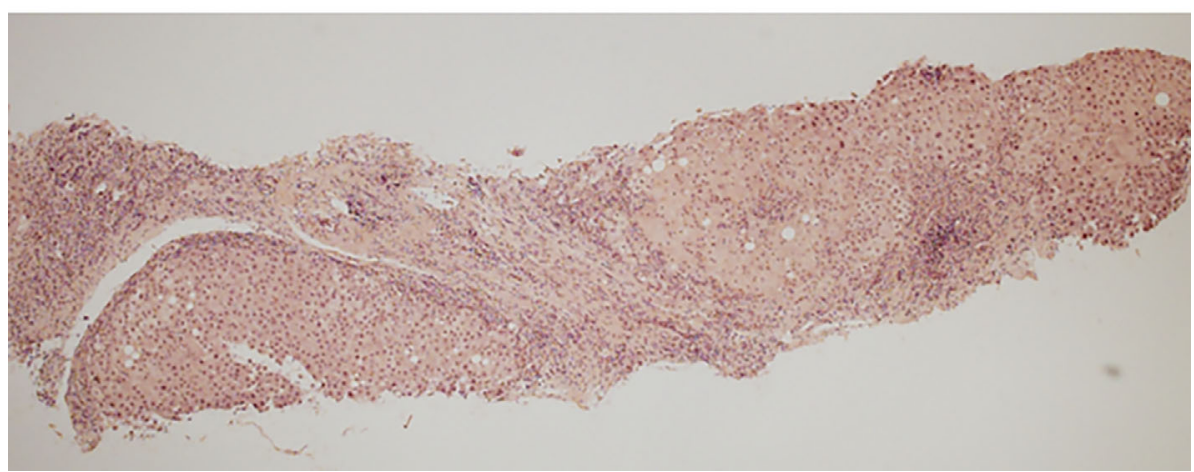

Figure 3. Histological examination of the liver 3 years after the initial diagnosis of NASH. Advanced fibrosis was found throughout the entire liver specimen. Lipid droplets decreased, consistent with burnout NASH (a, b). Congo red staining showed no deposition of amyloid (c). (a) Hematoxylin and Eosin staining, original magnifications: 10x, (b) Azan staining, original magnifications: 10×, (c) Congo-red staining, original magnifications: 10×. NASH: nonalcoholic steatohepatitis

Table 2. Plasma Osteopontin (OPN) Levels.

\begin{tabular}{lcccc}
\hline & $\begin{array}{c}\text { Other patients } \\
\text { with NASH } \\
\text { cirrhosis }(\mathrm{n}=3)\end{array}$ & $\begin{array}{c}\text { At the diagnosis of } \\
\text { NASH cirrosis } \\
\text { in present case }\end{array}$ & $\begin{array}{c}\text { After 2 years' treatment } \\
\text { of dexamethasone } \\
\text { in present case }\end{array}$ & $\begin{array}{c}\text { After SBP } \\
\text { in present case }\end{array}$ \\
\hline OPN $(\mathrm{ng} / \mathrm{mL})$ & $408 \pm 147^{*}$ & 1,970 & 838 & 1,930 \\
\hline
\end{tabular}

* The mean value of plasma OPN levels in three other patients with NASH cirrhosis at our hospital.

NASH: nonalcoholic steatohepatitis, SBP: spontaneous bacterial peritonitis

gesting that the hedgehog pathway had been activated in the latter $(11,16,17)$ (Fig. 4c and d). These results strongly suggest that OPN, which was probably induced by MM, participated in the progression of liver fibrosis in this patient.

\section{Discussion}

Over a period of 6-10 years, 5-20\% of patients with NASH progress to cirrhosis (2). Risk factors for fibrosis progression in patients with NASH include diabetes, obesity, age and high inflammation on initial liver biopsy sam- 
a

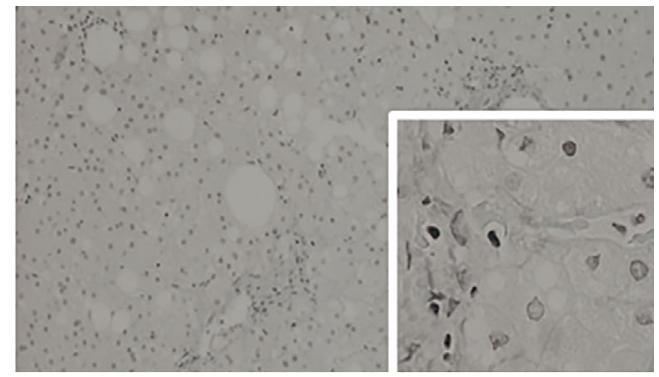

$\mathrm{b}$

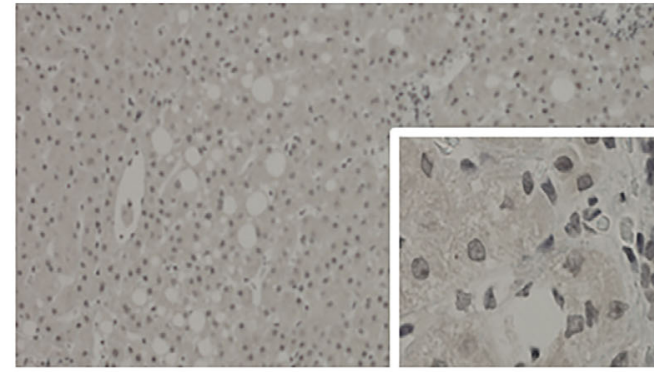

c

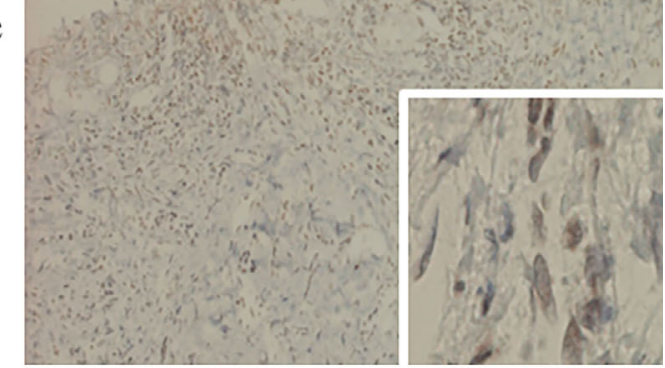

d

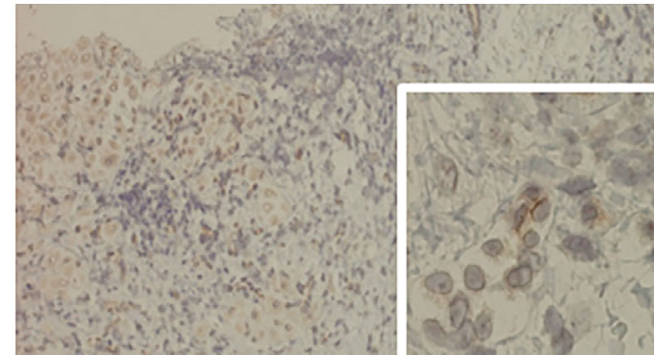

Figure 4. Immunohistological findings of the liver at the initial diagnosis of NASH (initial biopsy) $(a, b)$ and three years after the initial diagnosis of NASH (second biopsy) (c, d). (a, c)

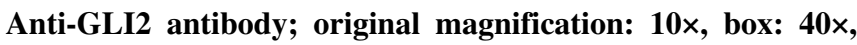
Nuclear GLI2 expression was found at the portal area of the liver at the second biopsy, but it was absent at the initial biopsy. (b, d) Anti-IHH antibody; original magnification: 10×, box: 40x. Cytoplasmic IHH was found at the portal area and periportal hepatocytes of the liver at the second biopsy, but it was absent at the initial biopsy. NASH: nonalcoholic steatohepatitis, IHH: Indian hedgehog protein

ples $(3,4)$. Our patient showed relatively rapid fibrosis progression. Although obesity had been present, her body weight did not increase after the initial diagnosis of NASH, and high inflammation was not found on the first liver biopsy.

We initially assumed that the disease progression had been caused by complications causing cirrhosis, such as autoimmune hepatitis, or a reduced liver function, such as amyloidosis. MM was diagnosed during systematic scrutiny after the diagnosis of NASH. NASH complicated by MM is extremely rare, with only one previous case report in the literature; that patient also developed burnout NASH cirrhosis (18). Factors common to NASH and MM include the expression of certain cytokines, including vascular endothelial growth factor, interleukin-6, and tumor necrosis factor$\alpha$ (18). The evaluation of similar patients may reveal further connections.

NASH-related cirrhosis is associated with the activation of the hedgehog pathway $(10,16)$. This pathway is typically silent in healthy livers but is activated when injury stimulates the production of hedgehog ligands, such as IHH (11). The activation of hedgehog signaling in liver cells promotes fibrosis by the conversion of hepatic stellate cells into myofibroblasts and by increasing OPN expression $(19,20)$. Plasma and hepatic OPN levels have been reported to be high in patients with NASH-associated advanced fibrosis $(10,21)$. Furthermore, OPN neutralization may prevent progressive hepatic fibrosis in NASH patients (10). Immunostaining of the liver tissue of our patient revealed the expression of GLI2 and IHH, markers indicating the presence of hedgehog-responding cells and the production of hedgehog ligand, respectively $(17,19)$. These results showed that the hedgehog pathway was activated in the liver of a patient with NASH complicated by MM.

In addition to its role in fibrosis progression, OPN has been reported to be important in bone resorption. OPN concentrations are reportedly higher in patients with MM than in those with monoclonal gammopathy of undetermined significance or healthy controls $(5,6,8,9)$. Furthermore, OPN levels have been reported to increase with the progression of MM (5, 8, 9). Although MM in our patient was not very advanced and she had no bone lesions, her plasma OPN level was high. In patients with MM, OPN is produced by osteoclasts, especially directly by plasma cells $(6,7)$. OPN levels were shown to increase with the progression of MM $(1,822 \pm$ $299,776 \pm 160$ and $309 \pm 34 \mathrm{ng} / \mathrm{mL}$, in MM with bone lesions, MM without bone lesions and healthy individuals, respectively) (8); however, this connection remains controversial (7). In the present case, the plasma OPN level was markedly higher than that of patients with NASH cirrhosis (408 $\pm 147 \mathrm{ng} / \mathrm{mL}$, Table 2). This increase in OPN may have been due to the synergistic effects of MM and activated stellate cells in NASH, probably due to its production by plasma cells.

Taken together, these findings suggest that the MMderived increase in OPN may have been responsible for the rapid progression of fibrosis in our patient. She did not receive aggressive treatment for MM because of her liver dysfunction and poor condition. However, more aggressive treatment may have prevented the progression of fibrosis by stopping the increase in OPN. Regarding limitations associated with this study, we were unable to identify the hedgehog-responding cells or OPN-overexpressing cells from samples of liver biopsies. However, the expression pattern of hedgehog ligand or GLI2 was similar with those reported previously (11). In this report, the hedgehog- 
responding cells were reported to be liver progenitor cells and stromal cells (11). Further investigations into the mechanism underlying how the hedgehog pathway was activated in this case are required.

In conclusion, we encountered an unusual patient with NASH, who showed rapid fibrosis complicated by MM. OPN due to MM may synergistically contribute to fibrosis progression in patients with NASH.

The authors state that they have no Conflict of Interest (COI).

\section{References}

1. Angulo P. Nonalcoholic fatty liver disease. $N$ Engl J Med 346: 1221-1231, 2002

2. de Alwis NM, Day CP. Non-alcoholic fatty liver disease: the mist gradually clears. J Hepatol 48 (Suppl): S104-S112, 2008.

3. Adams LA, Sanderson S, Lindor KD, Angulo P. The histological course of nonalcoholic fatty liver disease: a longitudinal study of 103 patients with sequential liver biopsies. J Hepatol 42: 132-138, 2005.

4. Argo CK, Northup PG, Al-Osaimi AM, Caldwell SH. Systematic review of risk factors for fibrosis progression in non-alcoholic steatohepatitis. J Hepatol 51: 371-379, 2009.

5. Sfiridaki A, Miyakis S, Pappa C, et al. Circulating osteopontin: a dual marker of bone destruction and angiogenesis in patients with multiple myeloma. J Hematol Oncol 4: 22, 2011.

6. Minarik J, Pika T, Bacovsky J, Petrova P, Langova K, Scudla V. Prognostic value of hepatocyte growth factor, syndecan-1, and osteopontin in multiple myeloma and monoclonal gammopathy of undetermined significance. ScientificWorldJournal 2012: 356128, 2012.

7. Standal T, Hjorth-Hansen H, Rasmussen T, et al. Osteopontin is an adhesive factor for myeloma cells and is found in increased levels in plasma from patients with multiple myeloma. Haematologica 89: 174-182, 2004.

8. Saeki Y, Mima T, Ishii T, et al. Enhanced production of osteopontin in multiple myeloma: clinical and pathogenic implications. Br J Haematol 123: 263-270, 2003.

9. Valkovic T, Babarovic E, Lucin K, et al. Plasma levels of osteopontin and vascular endothelial growth factor in association with clinical features and parameters of tumor burden in patients with multiple myeloma. Biomed Res Int 2014: 513170, 2014.

10. Syn WK, Choi SS, Liaskou E, et al. Osteopontin is induced by hedgehog pathway activation and promotes fibrosis progression in nonalcoholic steatohepatitis. Hepatology 53: 106-115, 2011.

11. Swiderska-Syn M, Suzuki A, Guy CD, et al. Hedgehog pathway and pediatric nonalcoholic fatty liver disease. Hepatology 57: 1814-1825, 2013.

12. Brunt EM, Janney CG, Di Bisceglie AM, Neuschwander-Tetri BA, Bacon BR. Nonalcoholic steatohepatitis: a proposal for grading and staging the histological lesions. Am J Gastroenterol 94: 24672474, 1999.

13. Kleiner DE, Brunt EM, Van Natta M, et al. Design and validation of a histopathological scoring system for nonalcoholic fatty liver disease. Hepatology 41: 1313-1321, 2005.

14. Durie BG, Salmon SE. A clinical staging system for multiple myeloma. Correlation of measured myeloma cell mass with presenting clinical features, response to treatment, and survival. Cancer 36: 842-854, 1975.

15. Greipp PR, San Miguel J, Durie BG, et al. International staging system for multiple myeloma. J Clin Oncol 23: 3412-3420, 2005.

16. Guy CD, Suzuki A, Zdanowicz M, et al. Hedgehog pathway activation parallels histologic severity of injury and fibrosis in human nonalcoholic fatty liver disease. Hepatology 55: 1711-1721, 2012.

17. Jung Y, McCall SJ, Li YX, Diehl AM. Bile ductules and stromal cells express hedgehog ligands and/or hedgehog target genes in primary biliary cirrhosis. Hepatology 45: 1091-1096, 2007.

18. Nakahara T, Utsugi $T$, Ohyama $Y$, et al. Type 2 diabetes mellitus complicated with smoldering myeloma and non-alcoholic steatohepatitis. Intern Med 44: 838-842, 2005.

19. Omenetti A, Choi S, Michelotti G, Diehl AM. Hedgehog signaling in the liver. J Hepatol 54: 366-373, 2011.

20. Das S, Harris LG, Metge BJ, et al. The hedgehog pathway transcription factor GLI1 promotes malignant behavior of cancer cells by up-regulating osteopontin. J Biol Chem 284: 22888-22897, 2009.

21. Nagoshi S. Osteopontin: versatile modulator of liver diseases. Hepatol Res 44: 22-30, 2014.

The Internal Medicine is an Open Access article distributed under the Creative Commons Attribution-NonCommercial-NoDerivatives 4.0 International License. To view the details of this license, please visit (https://creativecommons.org/licenses/ by-nc-nd/4.0/).

(C) 2018 The Japanese Society of Internal Medicine Intern Med 57: 2013-2018, 2018 